\title{
Theories in the Light of Contingency and Change: Possible Future Worlds and Well-Grounded Hope as a Supplement to Truth
}

\author{
Ulrich Frank \\ University of Duisburg-Essen \\ ulrich.frank@uni-due.de
}

\begin{abstract}
Based on a critical account of the dominant concept of theory, the paper presents an alternative, wider notion of theory. It is motivated by the need to cope with a contingent research subject and the assumption that IS should provide an orientation for managing the digital transformation. Unlike neopositivistic notions of theory, the proposed conception is not restricted to descriptions of the factual, but may be aimed at designing possible future worlds. Conceiving of possible future worlds requires overcoming the barriers created by language that constitutes our idea of the present world. The paper discusses the resulting methodological challenges and outlines how they might be addressed.
\end{abstract}

\section{Introduction}

There is a phenomenal accomplishment, an outstanding document of human cognition, a source of inspiration, a rich reference to make sense of the world we live in, a powerful idea to structure our thoughts and to give us confidence. I am speaking about the concept of theory which is at the core of modern science. There are numerous examples of theories that have a substantial impact on everyday life; some of which we might not even be aware of, even though we depend on them. Others serve us as an explicit and ultimate model to explain unobvious occurrences and to reduce our uncertainty about the consequences of natural events and human action. While the benefits of theories in the natural sciences are widely agreed upon, there are serious concerns over the concept of theory: "If any problem in the philosophy of science justifiably can be claimed the most central or important, it is that of the nature and structure of scientific theories ..." ([1], p. 3). First, there is the problem of defining a concept of theory that permits a clear distinction of theories and other knowledge offerings. There are various properties that are often regarded as necessary, for example, the falsifiability postulate. However, those constraints are not sufficient for an unambiguous demarcation, since they do not, for example, permit a convincing answer to the question of whether a construction proposed as a theory cannot be reduced to a more general construction. Other concerns relate to the concept of causality as it is inherent to a widespread understanding of theory and reflected in the Hempel-Oppenheim schema [2]. The preferred, if not the only accepted, way to represent theories in the natural sciences is to formalize them. In the ideal case, a theory is a model (an interpretation) of an axiomatic system. Hence, the precision or objectivity of a theory is mainly challenged by the question of how terms that are introduced by a theory relate to terms of a theoryindependent observation language [3]. As a consequence, sophisticated concepts of theory were proposed to avoid or mitigate this problem, such as the "semantic" [4] or the "non-statement" view [5].

However, despite its intensity, the debate in philosophy of science hardly unsettled natural scientists. Independent of the lack of a precise definition, the idea of a theory seems to work and brings exciting results. The situation is clearly different in the social sciences in general, and in IS in particular. While neo-positivistic research methods are predominant, there are serious concerns over the adequacy of this paradigm for the social sciences. The concerns, which are related to principal ontological and epistemological presuppositions (see, e.g., [6], [7]), include challenging the role of theory. Avison and Malaurent complain about the reference to theories being regarded as an obligatory property of a contribution to qualify as academic ("theory fetish", [8]). Various other authors regard focusing on theories or, more likely on theory testing, in IS as an inhibitor of inspiring research results. Some doubt that the theories used in IS are of any value for decision makers in practice, a topic of intense controversy in the notorious "rigor versus relevance" debate [9], [10].

In this paper, I will first argue that the compulsive use of an inappropriate idea of theory contributes to 
the parlous state of the field. Then I will propose a notion of theory that accounts for the peculiarities of IS. It combines (semi-) formal conceptual models with thought provoking narratives. With respect to justification, it suggests supplementing truth with grounded hope.

\section{Theories in IS: Signs of (Mis-) Concep- tion}

It seems that the debate on research methods in general, and the use of theories in particular, is not more than background noise to the ongoing reproduction of what is taken as common wisdom by many: that scientific research "should be based on a set of well-defined hypotheses, unbiased and reproducible procedures for collecting evidence that supports or refutes the hypothesis, and sound analytical procedures for drawing appropriate conclusions from the evidence." (MISQ, Sept. 1989, editorial statement). Nevertheless, I am naive enough to launch a further attempt to challenge the dominant model of research in IS.

\subsection{Inconsistencies}

The quest for a theoretical foundation is top of the list reviewers use to assess papers. However, not only are there are "conflicting notions of theory" [11], but also a lack of definitions that could clearly distinguish theories from other knowledge offerings. Even though this may be seen as an unavoidable reflection of the complexity of the subject, it indicates a questionable lack of consistency in the foundation of a discipline that regards the quest for rigor as one of its cornerstones.

The dominant conception of theory in IS seems to correspond to the behaviorist model, which, for the sake of simplicity, I will refer to as neo-positivistic. It is characterized by adopting ideas from logical positivism, such as the Hempel-Oppenheim schema, and by following Popper in respect of the belief that testing theories by confronting hypotheses deduced from them with reality is a key element of scientific inquiry. However, in contrast to Popper's demands, the information content of models of theories tested in IS is often systematically reduced, sometimes close to tautology, to avoid immediate refutation. This does not come as a surprise, since there are no theories that would satisfy the standards of theories in the natural sciences. Furthermore, theory testing is not aimed at refutation in the strict sense that Popper had in mind. Instead, testing is usually aimed at applying statistical procedures to calculate confidence levels. The fact that Popper regarded assigning probabilities to propositions as unscientific does not necessarily disqualify this approach. However, it is odd that this conflict is usually ignored and that, in general, the epistemological relevance of statistical procedures remains undisputed.

\subsection{Contingency and Change}

Theories used in IS lack one essential property of impressive theories in the natural sciences. They do not allow for prediction. In part driven by this fact, there has been an intensive debate in the social sciences and in philosophy of science whether or not it is appropriate to apply the same model of research and, hence, the same concept of theory to both the natural sciences and the social sciences. I will not go into the details of this debate, but look at one common argument used by neo-positivists to justify behaviorist methods. That argument holds the lack of powerful theories does not indicate the principal unsuitability of the behaviorist model for the social sciences, because it is only a matter of time until we see more powerful theories. While the first part of the argument cannot be invalidated for logical reasons, the second can be challenged - and I think it should be challenged because of its potentially harmful effects. Among a plethora of counter-arguments I will focus on three that are of special relevance for our field. The first argument is an ontological one and reflects the assumption that action systems are characterized by contingency: actions may follow certain patterns, but they do not have to. Our experience in everyday life as well as numerous studies in sociology and social psychology confirm this assumption.

The second argument is based on a common idea of man that is also shared by neo-positivists like Popper. According to this idea, human beings have free will. If we refuse to abandon this comforting idea, theories with the predictive power of those in the natural sciences are not conceivable in the social sciences: everyone can decide to follow the pattern described in a theory or not. The third and strongest argument focuses on a phenomenon that is at the core of our research subject: change and the role of language. While we do not yet know the details of the digital transformation, there are good reasons to assume that it will fundamentally change the way we work, live, and think. Therefore, it seems a core responsibility of a discipline that is supposed to study the use of digital technologies in organizations to develop insights and guidelines that help benefit from the forthcoming change instead of suffering from it. Putting aside the question how that could be achieved, it is obvious that the neo-positivistic para- 
digm is not suited to coping with this challenge. This is for two interrelated reasons. First, behaviorist research is restricted to studying the present or, more likely, the past. According to that doctrine, theories that were successfully tested in the past could be used to forecast the future or, at least provide guidance for planning the future. However, lessons learned in the past are only of limited use for creating the future in an era of disruptive change. They may teach us what we do not want, but are insufficient to show us what future we can aim for. Second, the world as we perceive it, depends on the language we speak, in other words: "...there is no other kind of cognition than through concepts." ([12], p. 202). Therefore, we can think of the world and especially of social systems as being constructed through language [13] and speech acts [14]. Hence, social, economic and technical change is always accompanied by changing the relevant language. At the same time, software systems are linguistic constructions, too. Therefore, the construction of information systems will have an impact on the language their users speak and subsequently, those altered language games will have an impact on the design of future software systems. This subtle pattern of mutually re-enforcing change has been known for some time, and it has a tremendous impact on the research that is required to provide guidance for the digital transformation. If the modification of language is an essential characteristic of this transformation, we need to address the question of how the language we speak and the language we use to build information systems should be altered to prepare for a better future. This corresponds to Rorty's pragmatic view of language: "Philosophers have long wanted to understand concepts, but the point is to change them so as to make them serve our purposes better." ([15], p. 25). If IS takes the challenge of the digital transformation seriously, it can neither neglect the essential role of language (both of natural languages and implementation languages) nor the need to analyze how languages should look to sow the seeds of a better future. Here, the neo-positivist paradigm hits its limits. First, the idea of formulating "objective" theories would be shattered, because theories as linguistic constructions would always reflect the reality or ontological practices ([6], p. 7) of a certain time frame. Second, developing new concepts to foster better information systems and in general, a more desirable world, would make the correspondence theory of truth and, hence, empirical testing procedures widely redundant: the new concepts and action systems they refer to are deliberately made different from the current reality. Design Science [16], which has achieved a modest popularity in recent years, does not provide a convincing founda- tion for this kind of research. Its core assumptions are positivistic (design objectives and design theories should be grounded empirically) and the idea of construction it promotes reflects a mechanistic worldview (searching for solutions in a design space) (for a more detailed critique of Design Science see [17]).

\section{A Multifaceted Conception of Theory}

Thus far, our considerations have resulted in two main insights: First, the neo-positivistic idea of theory is not suited as a leading paradigm of research in IS. Second, there is need for a conception of theory that accounts for the peculiarities of our research subject, in particular for the role language plays in the digital transformation.

\subsection{Theories as Multiple Constructions}

The conception of theory that I will briefly introduce now is based on two assumptions: First, it is not possible to define a concept of theory that would allow for an unambiguous distinction of theories from other knowledge contributions. However, that does not mean to give up on this distinction, because that meant to abandon the claim for the superiority of scientifically approved knowledge. Therefore, criteria are required that support making a corresponding decision. Second, theories cannot be appropriately conceptualized, if they are reduced to mere linguistic constructions. Against this background I propose that any knowledge offering qualifies as theory as long as it satisfactorily fulfills essential epistemological postulates with respect to a certain methodological and cultural context (for a more detailed discussion see [17]). Three epistemological postulates seem to be pivotal: abstraction, originality, and justification.

According to the neo-positivistic notion of theory, abstraction may reflect the classification of objects found in the factual world. I suggest extending the scope of abstraction by intentionally going beyond the factual to target possible future worlds. This is not an entirely new facet of theory, but is in line with the original meaning of the Greek " $\Theta \varepsilon \omega \rho i ́ \alpha "$, which literally means "outlook" in the sense of looking beyond the obvious. At the same time, it is similar to studies on the future [18], [19], but different from those with respect to focusing on the construction of possible futures. From a more philosophical perspective, this suggests also aiming for detachment and transcendence. In other words, a theory should reflect a picture of what we could see, if we overcame the restrictions of our physical, social, and political exist- 
ence; and, hence, of our language. Developing possible future worlds must not be mistaken for science fiction. It makes sense only if it satisfies certain demands related to justification (see 4.3).

Originality comprises two essential aspects. Original knowledge should be new, that is, not yet known, and it should satisfy the superiority postulate, which means it should in some respect be superior to existing knowledge. In other words, it should be capable of surprising those peers who work in the same field. This could also be achieved by shattering accepted knowledge. Since justification creates a particular challenge especially with respect to the construction of possible future worlds, we shall look at this issue in more detail in 4.3.

The epistemological quality of a knowledge offering also depends on its documentation: any idea qualifies as theory only if it is externalized in a way that supports its evaluation and use. In other words, for theories to exist they need to be mediated in a way that makes sense. Among other aspects, documentation implies the use of an elaborate, precise language. Last, but not least, enacting or effectively constructing a theory requires a conception of a scientific community, which is characterized through a commitment to a certain culture and a range of accepted methodological practices (see 4). This context is essential for deciding whether or not the epistemological postulates are satisfied. In the remaining part of this section, we will look at two kinds of knowledge contributions that are of specific relevance for the design of possible future worlds, where advanced information systems are supposed to play a pivotal role.

\subsection{Conceptual Models}

Models have always been at the core of scientific inquiry. They serve to describe the subject of investigation in a way that enables further analysis, and allows for comparison and transformation. Furthermore, they provide a medium for communication, and moreover, models are the only instrument available to develop an idea of the future. Using the metaphor of a map, Wood expresses the pivotal role of models for our cognition clearly: "And this, essentially, is what maps give us, reality, a reality that exceeds our vision, our reach, the span of our days, a reality we achieve no other way." ([20], p. 4 ff.).

While models are essential for imagining the future in general, a specific kind of model is of particular relevance to the digital transformation. Software will have an ever increasing impact on shaping future worlds. For the (re-) use of software systems, for integrating software systems and for adapting them, the underlying conceptual models are highly relevant. Conceptual models are aimed at bridging the gap between the natural language in the relevant domains of discourse and the implementation language of choice, which, in most cases, will be an objectoriented programming language. The bridging function is achieved by using foundational/ontological concepts for modeling languages that are used in both natural language and implementation languages, such as class, object, and attribute. A conceptual model that serves this purpose cannot be described with natural language, because natural language is vague and ambiguous. In addition, preparing for automation may require the modification of concepts, such as the concept of a document. Finally, the design of software systems will often be aimed at flexibility, integration and reuse. That requires powerful abstractions which in turn may suggest the introduction of concepts which do not have a direct counterpart in natural language. Hence, conceptual models are reconstructions of concepts found in natural language domains. These reconstructions are created with modeling languages; either a general purpose modeling language (GPML) like the UML or a domainspecific modeling language (DMSL).

Developing conceptual models that can serve as foundation for a range of present and future software systems poses a considerable research challenge. How are they related to theories? Conceptual models may satisfy the abstraction postulate, if they are not restricted to a particular organization, but come with the claim to cover a wider range of use cases, as intended with reference models. They may also account for originality, if the systems they enable provide important, non-trivial features that were not known of before. Unlike the positivistic notion of theory, conceptual models do not necessarily aim to represent factual domains. They may also serve to create an image of a possible future domain, or, in other words: to propose a language to conceive of this future domain. Such a language may look more or less familiar to prospective users, depending on how radical the intended change is supposed to be. Also, they serve as a foundation for corresponding software systems. For this purpose, there should be transformation rules that define how to map the concepts of a modeling language to those used in the targeted domain. Research on conceptual models does not only comprise the construction of models, but also the design of new modeling languages that provide more powerful abstractions or that are better tuned to the requirements of specific domains.

By focusing on conceptual models and modeling languages, IS research goes beyond contributing 
directly to the design of possible future information systems. Conceptual models also serve to illustrate possible future action systems. The ever increasing penetration of information systems into organizations advances the case for models that integrate action systems with information systems through the use of common concepts. This would be similar to what is already done in the field of enterprise modeling [21]. However, it would be clearly more radical in the sense that it would target delivering models that overcome the artificial border between the language games of IT and of the business.

\subsection{Narratives}

While conceptual models can serve as a powerful foundation for creating possible future worlds, they are not sufficient. To assess a possible world, people need to be able to imagine how it would be to live in that world or, in other words, what sense such a world would make. While conceptual models are clearly better suited to serve this purpose than mathematical models or code fragments, they intentionally fade out all aspects of the intended use context that bulk against formalization, such as symbolic action, informal communication, and power games. This can be supported by narratives of a possible future. Those narratives would aim at rich descriptions of certain aspects of life in a future world. They could be related to illustrate new practices enabled by new technologies. Narratives might supplement conceptual models or stand on their own.

While I agree with Rorty that good novels and films can tell us more about life than mediocre science, I would not support his idea of a "general turn against theory and toward narrative" ([22], p. xvi), and I would not want to abandon the epistemological postulates outlined above. I prefer to think of scientifically grounded narratives, which have similarities to hermeneutic conceptions of theory. They also resemble the concept of the theory of situated practice proposed by Hovorka et al. who stress the use of narratives to foster "comprehending the world" by providing access to "the background of the worlds in which people conduct their day-to-day practices." ([23], p. 16) However, the scientifically grounded narrative concept can differ from those conceptions in that it does not primarily aim to contribute to understanding the factual world (even though that should be a side effect), but to comprehend possible future worlds. That implies that the hermeneutic demand to get involved cannot be satisfied directly, because the practices that would make it possible to do so do not exist. Nevertheless, that would not mean to give up the demand for comprehension and empathy ([24], p. 20) nor for sense-making [25]. Such a narrative should present a differentiated picture that includes possible conflicts, inconsistencies and threats. In addition, it should of course be made clear that narratives of this kind address a contingent matter: the future they describe should be possible, but that neither means that it will become reality, nor that there is a deterministic way to achieve it.

\section{Methodological Considerations}

If research wishes to develop ideas of possible worlds that may serve as an orientation for change, traditional research methods, which are mainly focused on empirical evidence or on existing phenomena, are not sufficient. Below I will first present two facets of a corresponding research method, before examining approaches to justification.

\subsection{The Role of Contradictions}

Apart from the essential question of how we want to live and work tomorrow, the design of possible future worlds is also guided by goals that, while largely undisputed, are hard to achieve. To give a few examples: Information systems should be integrated with the action systems they are supposed to support. Software artifacts should be reusable to reduce development costs and improve quality simultaneously. Software systems should feature a high level of integrity of the data they manage. Software systems should be adaptable without jeopardizing a system's integrity. Organizational rules should be clearly defined to ensure the reliability of the services offered to customers. Organizations should be flexible to cope with external threads and opportunities.

While each of these demands seems suited to serve as an orientation for improving the factual world, a closer look at the measures required to achieve them reveals that some are in conflict or even contradictory. To take a closer look at one example only: the idea of integrity, both with respect to action systems and to software systems, demands the existence of a comprehensive schema that can be referred to whenever an activity needs to be performed. Flexibility on the other hand requires systems to be able to cope with changing requirements. Therefore, in clear contradiction to the demand for integrity, the quest for flexibility would require the introduction of less restrictive constraints that allow for a range of different interpretations.

Contradictions of this kind are well known. They are either treated as trade-offs or intentionally ig- 
nored by design guidelines such as loose coupling, service-oriented architectures, or agile organizations. However, if we do not take those contradictions for granted, but ask what would be needed to relax or overcome them, we could accomplish progress in a convincing sense: being able to achieve goals everybody agreed on without compromising them with trade-offs. My experience with designing software systems and working on organizational theory suggests that focusing on contradictions like those above can be frustrating, but may also inspire us to transcend the frame we used to conceptualize the subject of our work.

\subsection{Destruction and Liberation}

If we accept the presupposition that the world as we see it is the reflection of a linguistic construction (that does not mean that it would not exist without language), change is always accompanied by a change of concepts. To give just one example: The concepts of enlightenment eventually changed the language we use to speak and think about politics. But how might a method that guides the design and assessment of new concepts look? A moderate version of such a method is an inherent part of any serious scientific study. The concepts that are used to describe the phenomenon of an investigation will usually undergo a critical analysis aiming to discover the likes of ambiguities, inconsistencies, and hidden value judgments. After unmasking a concept, it would then either be abandoned as an unsuitable instrument or replaced by a refined/reconstructed version. However, this kind of critical analysis will usually happen within a given language paradigm. Conceiving of possible futures requires more radical procedures, the establishment of which is a tremendous, if not frightening, challenge. Language is a pivotal tool for we scientists. We have honed this tool through a long process of education and reflection, so that it now allows us to recognize things we did not see before, and to describe and solve puzzles that we were not even aware of in the past. At the same time, it delimits our world [26]. Especially, in the case of scientists it can be seen as a "web" of our "own creation" that we are trapped in ([27], p. 199).

There are various proposals in philosophy advising how we might free ourselves from this trap. Nietzsche uses the metaphor of a child to illustrate what he regards as the ultimate development phase of the human spirit, a phase where we get rid of the chains created by a language that was imposed on us and start to develop our own language: "Innocence the child is and forgetting, a beginning anew, a play, a self-propelling wheel, a first movement, a sacred Yea-saying." ([28], p. 24). How could we become as free as the child in Nietzsche's allegory? The phase that precedes the child is the lion, that is a phase of rebellion, of questioning the world and the language we were socialized with. Apart from the radical essentialist sense of such an endeavor, it corresponds clearly to the critical method that we all know.

Heidegger tried to develop an ontology that reflects the foundational aspects of being. In doing so he attempted to overcome those concepts previously used to convey ideas of what being is by inventing a new vocabulary that served as a tool for uncovering formerly hidden aspects of human life and action [29]. Derrida suggested an approach he called déconstruction [30], which, while it could be regarded as a method to analyze text by challenging/resolving the concepts it is based on, Derrida refused to call a method, because method is a key concept of the established scientific terminology, which he wanted to overcome. To enable the deconstruction of concepts, he introduced new concepts. Text represents any form of linguistic expression or communicative action. The made-up word différance expresses both the obligatory function of semantic differences to enable communication and the impossibility of clearly defining differences in meanings.

What can we learn from philosophers who tried to develop new languages? First, they teach us that new concepts are likely to alienate others. Reading Heidegger or Derrida is painful for most of us and will be regarded as annoying and pointless by many. Second, trying to overcome the limitations of our language is an important prerequisite not only for seeing the world through other eyes, but also for conceiving of a possible future that is constituted by a language yet to emerge. Third, such an undertaking is extremely risky, because it means dissolving the floor beneath our feet as we walk. Derrida expresses this ambivalent effect of deconstruction as follows: "The future can only be anticipated in the form of an absolute danger. It is that which breaks absolutely with constituted normality and can only be proclaimed, presented, as a sort of monstrosity." ([30], p. 5).

What does the idea of deconstruction mean for IS research? It does not mean recommending the introduction of jargon á la Heidegger or Derrida, and nor does it mean systematically destroying the terminological foundation of the discipline. It also does not imply systematically replacing the language we use to describe and analyze the subject of our research with another. Instead, I think of it as a supplement to our regular business. In order to develop a critical appreciation of the digital transformation, we need to focus on the role of language. Doing so involves 
maintaining a critical stance toward the steady stream of new terms and buzzwords that are introduced to convey supposed ideas of progress. Instead of adopting those concepts, we should instead aim to unmask them and to develop new concepts that are better suited to represent emerging phenomena. Only if we cease to regard the concepts underlying spreadsheets, operating systems, or accounting systems as given can we think of alternative options. Only if we do not take the semantics of current modeling and implementation languages for granted will we be able to think of abstractions that might be suited to build systems that cope better with conflicting requirements, and that are simultaneously better prepared for a contingent future. Most of us will regard the concept of a business process as crucial for our field. However, if we want to envisage innovative forms of collaboration, we probably need to give up this concept or renovate it by deconstructing it. It is needless to mention that a discipline that approaches its subject with a critical, deconstructivist claim will be credible only if it develops possible future images of its own by challenging and eventually deconstructing the concepts the game called IS based upon.

This kind of critical deconstruction and reconstruction would not only help to conceive of a future that will be constituted by a language yet not known, it would broaden our perspective on the current world: "All formation of new concepts, all change in concepts, involves discovery of the world - that is, the development of a new way of looking at the world ... which may be more or less borne out as time goes on. Every theory of formation of new concepts is also about discovering the way the world is." ([31], p. 34)

\subsection{Justification as a Challenge}

Suggesting a conception of theory that comprises models and narratives of possible future worlds may sound bizarre to some, because it seems to contradict the idea of science we are all used to; that however, is not my intention. Conceptual models and narratives could qualify as theories only if they are regarded as sufficiently fulfilling the essential epistemological postulates. However, the justification postulate creates a serious challenge. Abandoning the justification postulate would be a frightening option, because it would mean sacrificing one of the key achievements of modern science, the replacement of ideology and force with rationality.

Scientific inquiry typically asks why something (an object, a process, a phenomenon) is. An attempt to answer this question would typically be justified by showing that it is true for the time being. Conceptions of truth [32] are the pivotal criterion for justifying scientific claims. Apparently, a possible world cannot be shown to be true in the sense of the correspondence theory of truth, and even less so a new language. How might a procedure that serves to justify appropriateness or desirability look? The justification of possible future worlds comprises two main aspects: feasibility and attractiveness. Providing a satisfactory justification of both aspects would result in what we could call scientifically grounded hope. A procedure claiming feasibility will normally need to satisfactorily answer two questions, the first on whether certain aspects of a possible future world could be realized, and the second on whether there are aspects that are impossible to realize. To give an example of the first case: it might be possible to show that a conceptual model can be transformed into an executable representation. In any case, the transparency postulate requires making explicit all underlying presuppositions that should be true, if a transformation is possible or impossible. However, not every aspect of feasibility is invariant. Skills, attitudes, and common practices can change, as can the representation of information and the world in general. Therefore, scholars must investigate why the truth value of those presuppositions can change over time. In other words, they are not necessarily invalid, if it is shown that they do not fully apply to a present world.

While the idea of possible future worlds does by no means imply any kind of prescription, it will be necessary to evaluate the attractiveness of a possible future world to support those who may consider using it as an orientation. This kind of evaluation requires accounting for possibly unpleasant effects and conflicting goals. There is one main way of justifying the attractiveness of a possible future world in comparison to other alternatives: a discursive approach that attempts to account for all relevant aspects and for all interests involved. Such a discourse would have to be reflective in the sense that it would have to include a critical account of values, convictions, and of the language of the participants. During the course of a discursive evaluation, individual predispositions may be subject to change. A related point is that the participants would have to cope with having to evaluate a future world, which is characterized by a language they are not yet familiar with, using the concepts of the present world. The fewer commonalities between the two languages, the more likely it is that the attempt to develop a satisfactory evaluation of a possible future world will fail ([22], p. 9).

A discursive evaluation may be restricted to a relatively short meeting involving just a few participants, but it is more likely to happen over a longer 
period with many different contributions, and it does not have to result in a unanimous decision. Organizing such a process demands rethinking current patterns of academic communication. First, those are typically not discourse-centered, but focused on publications. Second, they exclude those who are not part of a specific scientific community. With respect to the complexity and relevance of the digital transformation, it may be more appropriate to widen the range of participants to include representatives of other disciplines and also people who are not associated with academic institutions. While I have considerable sympathy with a possible future that is guided by the core values of enlightenment, those are certainly not suited to providing clear guidelines, since they are subject to varying interpretations. Rorty suggests starting with an apparently small common denominator by striving for a future without (avoidable) pain [22]. If we accept this goal for all human beings, it implies a sense of empathy and solidarity, and finally one is in the middle of the grand political discourse of western civilization in general, of the political role of science in particular. While one does not have to agree with Rorty's proposal or any other vision of a better world, we can hardly tune out the discussion about desirable properties of the future from the academic discourse.

\subsection{The Role of Scientific Culture}

The process of justification outlined above includes various problems. Apparently, the outcome will depend on the participants. What abilities and attitudes should those people have to qualify? Furthermore, a discourse requires certain rules that need to be followed by all participants to prevent a process being deemed unsatisfactory. However, those criteria and rules must not be defined precisely, because they need to be subject to adaptation, too. Therefore, the only option that is left that does not sacrifice the demand for justification is to emphasize the need for a specific, scientific culture.

The culture of science is mainly characterized by critique, freedom, language, and transparency. These features cannot be separated from originality, abstraction, and justification. However, they stress a different perspective and additional aspects, which are relevant for a suitable conception of science. Critique is essential for all schools of philosophy of science. Emphasizing the role of critique in scientific culture has a number of implications. A critique not only requires a skepticism of commonly accepted wisdom and research results, it must work on the basis that nothing is exempt from criticism-as long as the critique is backed by conceivable reason. Hence, a critique can be directed against research methods or, basic epistemological and ontological assumptions, as well as against the language used in a certain discipline. In order for a critique to be effective, it must not only be possible, but also appreciated: a critique should not be regarded as a personal attack, but as a contribution to the evolution of knowledge. The notion of a critique being a key driver of knowledge implies the idea of freedom. Freedom refers to the lack of dominion: everyone is free to express his or her opinion without the threat of sanction, as long as he or she is able to support that opinion with conceivable reason. In an ideal conception of science, there is no duress. No viewpoint is preferred over another by virtue of the position of those who hold it. There is only the "peculiarly forceless force of the better argument” (translated from [33], p. 52).

\section{Examples}

What does that mean for the concept of theory in IS? It suggests we would be wise not to restrict our view to the factual world. We should not look only at existing technologies and related patterns of use but should adopt a broader perspective based on the recognition that the construction of action systems and information systems is contingent in the sense that it could result in many different outcomes. Therefore, we should try to look beyond the facts that happen to form reality. In a more radical sense, this kind of outlook involves challenging apparently selfevident and commonly accepted concepts. The following three examples serve to illustrate how theoretical contributions of this kind might look.

The development of reusable and adaptable conceptual models requires the selection of a modeling language. GPMLs can be reused in a wide range of cases. However, their support for the efficient design of models is poor, because they are restricted to basic concepts such as class, process, and attribute. DSMLs, on the other hand, improve productivity by providing modelers with domain-specific concepts such as goal, and organizational unit, but their range of reuse is limited. The approach presented in [34] shows that this conflict can be mitigated, if the language paradigm is changed. The work proposes a new language architecture that follows the idea of multilevel languages, that is, languages which allow for an arbitrary number of (meta) classifications. The language architecture is specified through a recursive meta-metamodel and can be instantiated to multiple domain-specific models. It not only allows the benefits of productivity and economies of scale to be 
combined, but also enables a new kind of information system that empowers users to modify a system on a conceptual level. This is achieved through a common representation of models and code, which in turn is enabled through a paradigmatic shift from traditional language architectures. Accordingly, the underlying language technology, which comprises a (meta) programming and modeling environment [35], is used in academic research exclusively, which makes empirical tests in the field almost impossible. The approach is evaluated by showing that the conflicts between undisputed goals can clearly be reduced.

Lanier develops a critical account of the current internet economy [36]. For this purpose, he identifies contradictions and related "absurdities" (Ibid., p. 19). He supplements his critique with two kinds of narratives. First, he uses narratives to illustrate possible excesses of the current trends. For example, he describes scenarios of new markets for customized drugs by using fiction to supplement his analysis of the technological and economic background. Second, he proposes a vision of what he calls "humanistic economics", which at its core is based on a new conception and implementation of the internet that promotes "network neutrality". It is aimed at overcoming "useless concentrations of wealth and power" (Ibid., p. 287). Like Lanier's work, van Reybrouck's book on possible future models of democracy is not only intended to contribute to academic debate, but targets a broader audience [37]. Even though it concerns a different discipline, namely political science, it may serve as an example of how to use narratives to address political aspects of change through the digital transformation. By illustrating contradictions ("paradoxa") produced by established forms of democracy, he concludes that if the principle ideas of democracy are to be preserved, it is necessary to overcome aspects of democratic institutions that we tend to take for granted. He starts with a critical account of the current situation that involves the discussion of empirical investigations and apparent phenomena, such as the problems of legitimacy and efficiency "western democracies are struggling with" (Ibid., p. 6). After an analysis of the causes of this unpleasant situation, he presents a new concept of democracy, which he calls "allotted assemblies". At its core, the notion suggests transferring certain political decisions from elected parliaments to councils and panels of citizens chosen by lot. Van Reybrouck offers two justifications for this proposal. First, he explains why assemblies might be suited to redress problems with the current models of democracy. Second, he refers to similar ideas by other authors as offering a precedent. He is aware of the fact that a suggestion that seems radical, if not bizarre to many, could not be implemented tomorrow. By pointing at examples from the past such as giving women the right to vote, he argues that "thinking further ahead" (Ibid., p. 131) can promote valuable change.

\section{Conclusions}

The neo-positivistic notion of theory has proven an extremely powerful construction. However, this is only the case when certain preconditions are fulfilled. Ignoring the preconditions and keeping the legend of "theory is king" alive would be unfortunate, because it promotes schematic scientific work and trivial results.

The conception of theory presented in this paper is intended to outline a possible alternative, not only for constructing and representing ideas, but also for organizing the process of academic inquiry. It accounts for general philosophical considerations and the peculiarities of the digital transformation as a pivotal research subject of IS. It corresponds to the original conception of theory ("looking beyond") and is in line with essential ideas of science. Nevertheless, some of the suggestions I made may be perceived as radical. There are certainly good reasons to challenge them, as there are good reasons to doubt the feasibility of satisfactory justification procedures. Furthermore, some might consider the reference to post-modernist philosophy beside the point. However, there is no doubt that research which is aimed at understanding the drivers of the digital transformation needs to account for the pivotal role of language. Furthermore, a discipline that desires to be more than a bystander or a follower (I am afraid that has largely been the case for the discipline in the past), needs to develop methods to conceive of possible future worlds and to make them a subject of inspiring discourses. Supplementing truth with justified hope for a better world [38] could be suited as a sense-making orientation for this kind of research.

\section{References}

[1] Suppe, F., ed., The structure of Scientific Theories, Univ. of Ill. Press, Urbana, 1974.

[2] Hempel, C.G. and P. Oppenheim, "Studies in the Logic of Explanation", Philosophy of Science, 15(2), 1948, pp. 135-175.

[3] Suppe, F., "Understanding Scientific Theories: An Assessment of Developments, 1969-1998", Philosophy of Science, 67, 2000, S102-S115. 
[4] Suppes, P., "What is a Scientific Theory?", in Philosophy of Science Today, S. Morgenbesser, Editor. 1967.

Basic Books: New York.

[5] Sneed, J.D., The Logical Structure of Mathematical Physics, Reidel, Dordrecht, 1979.

[6] Dreyfus, H.L., "Why Studies of human Capacities, modeled on Ideal Natural science, can never achieve their Goal", in Rationality, Relativism, and the Human Sciences, J. Margolis, M. Krausz, and R.M. Burian, Editors. 1986. M. Nijhoff: Dordrecht, Boston.

[7] Morgan, G., "Toward a More Reflective Social Science", in Beyond method: Strategies for social research, G. Morgan, Editor. 1983. Sage Publications: Beverly Hills, Calif.

[8] Avison, D. and J. Malaurent, "Questioning the theory fetish in information systems", Journal of Information Technology, 29(4), 2014, pp. 327-336.

[9] Lee, A.S., "Rigor and Relevance in MIS Research: Beyond the Approach of Positivism Alone", MIS Quarterly, 23(1), 1999, pp. 29-34.

[10] Chatterjee, S., "Information Systems Research and Relevance", Communications of the AIS, 6, 2001, pp. 1-7.

[11] Markus, L.M., "Maybe not the king, but an invaluable subordinate: a commentary on Avison and Malaurant's advocacy of 'theory light' IS research", Journal of Information Technology, 29(4), 2014, pp. 341-345.

[12] Kant, I., Critique of pure reason, Cambridge Univ. Press, Cambridge, 1998.

[13] Berger, P.L. and T. Luckmann, The social construction of reality: A treatise in the sociology of knowledge, 1st edn., Doubleday, Garden City, N.Y, 1966.

[14] Searle, J., Speech Acts, Cambridge University Press, Boston, 1969.

[15] Rorty, R., Rorty and his critics, 1st edn., Blackwell, Oxford, 2000.

[16] Hevner, A.R., S.T. March, J. Park, and S. Ram, "Design science in information systems research", MIS Quarterly, 28(1), 2004, pp. 75-105.

[17] Frank, U., Towards a Pluralistic Conception of Research Methods in Information Systems Research, ICB Research Report (7), University of Duisburg-Essen 2006.

[18] Chiasson, M., O. Henfridsson, H. Karsten, and J.I. DeGross, eds., Researching the Future in Information Systems: IFIP WG 8.2 Working Conference, Turku, Finland, June 6-8, 2011, 2011.

[19] Blass, E., "Researching the future: method or madness?", Futures, 35(10), 2003, pp. 1041-1054.
[20] Wood, D., The Power of Maps (Mappings: Society, Guilford Pubns, 1992.

[21] Frank, U., "Multi-Perspective Enterprise Modeling: Foundational Concepts, Prospects and Future Research Challenges", Software and Systems Modeling. 2013.

[22] Rorty, R., Contingency, irony, and solidarity, Cambridge University Press, Cambridge, New York, 1989.

[23] Hovorka, D.S., R.B. Johnston, and K. Riemer, Reclaiming Meaning in IS Theory: Theory for Why, not What: Information Systems Foundation Workshop, Australian National University, 2014.

[24] Wright, G.H. von, Explanation and Understanding, Cornell University Press, Ithaca, NY, 1971.

[25] Weick, K.E., Sensemaking in organizations, 2nd edn., Sage, Thousand Oaks, 1995.

[26] Wittgenstein, L., Tractatus logico-philosophicus, Routledge \& Paul; Humanities Press, London, New York, 1961.

[27] Morgan, G., Images of Organization, Sage, Beverly Hills, 1986.

[28] Nietzsche, F.W. and T. Wayne, Thus spake Zarathustra: A Book for Everyone and Nobody, Oxford University Press, Oxford, 2005.

[29] Heidegger, M., Sein und Zeit, 19th edn., Niemeyer, Tübingen, 2006.

[30] Derrida, J., Of grammatology, 1st edn., Johns Hopkins University Press, Baltimore, 1976.

[31] Schön, D.A., Displacement of concepts, Tavistock, London, 1963.

[32] Künne, W., Conceptions of Truth, Oxford University Press, Oxford, 2003.

[33] Habermas, J., Theorie des kommunikativen Handelns. Handlungsrationalität und gesellschaftliche Rationalisierung, Suhrkamp, Frankfurt/Main, 1981.

[34] Frank, U., "Multilevel Modeling: Toward a New Paradigm of Conceptual Modeling and Information Systems Design", Business and Information Systems Engineering, 6(6), 2014, pp. 319-337.

[35] Clark, T., P. Sammut, and J. Willans, Superlanguages: developing languages and applications with XMF, Ceteva, 2008.

[36] Lanier, J., Who owns the future? Simon \& Schuster, New York, 2013.

[37] van Reybrouck, D., Against Elections: A Case for Democracy, The Bodley Head, London, 2016.

[38] Rorty, R., Philosophy and social hope, Penguin Books, New York, 1999. 